\title{
AVALIAÇÃO ANTROPOMÉTRICA E POR BIOIMPEDÂNCIA DE UM PROGRAMA DE EDUCAÇÃO NUTRICIONAL PARA ESCOLARES NA FAIXA ETÁRIA DE 7-14 ANOS DURANTE O PERÍODO DE 10 MESES
}

\author{
A 10-MONTH ANTHROPOMETRIC AND BIOIMPEDANCE EVALUATION \\ OF A NUTRITIONAL EDUCATION PROGRAM FOR \\ 7 - TO 14-YEAR-OLD STUDENTS
}

\author{
Rubens Feferbaum ${ }^{1}$, Claudio Leone ${ }^{2}$, Rosemeire Casanova Nogueira ${ }^{3}$, Patricia Neri Cavalcanti ${ }^{3}$, \\ Eduardo Blanco Cardoso ${ }^{4}$, Monica Allende Serra ${ }^{5}$
}

\section{RESUMO:}

A prevalência do excesso de peso na infância aumentou nas últimas décadas. Considerando a relevância da escola na promoção da saúde, intervencões educativas individualizadas neste ambiente são necessárias. O presente estudo teve por objetivo determinar a influência de um programa de educação nutricional na promoção de hábitos alimentares saudáveis e no estado nutricional de alunos em idade escolar. Avaliaram-se 416 crianças e adolescentes, entre 7 e 14 anos, que frequentavam duas escolas públicas de educação complementar. A intervenção por 10 meses, realizada em uma das escolas, baseou-se na prática de atividades em educação nutricional destinadas a alunos, responsáveis legais e equipe escolar, com substanciais modificações do cardápio. Avaliou-se o peso, estatura, IMC, massa magra e massa gorda, nos grupos de intervenção (escola 1) e controle (escola 2 ). No início do estudo registrou-se respectivamente excesso de peso em ambas as escolas (34,0 e $28 \%$ ) e também de obesidade (16,9 e 10,4\%); com redução significativa desta última ao final do estudo $(p=0,001)$. Em relação ao crescimento dos alunos, este foi normal para a faixa etária nas duas escolas, sendo superior na escola $1(p=0,04)$. A composição corporal obtida por impedância bioelétrica registrou aumento da massa magra nas escolas 1 e 2 ( $p<0,01$ ), acompanhada de aumento da massa gorda apenas na escola $2(p=0,01)$. Estes resultados correlacionados à redução significativa do IMC, às proporções corporais, e ao crescimento estatural atingido pelos alunos da casa 1 , indicam que a alimentação adotada foi benéfica.

Key words: alimentação escolar, educação nutricional, composição corpórea em crianças e adolescentes.

\begin{abstract}
::
Over the past several decades, an increasing prevalence of excessive weight has been observed among children. Considering the importance of schools in promoting health, the implementation of individualized educational interventions in a school setting is necessary. The present study aimed to determine the influence of a nutritional education program in promoting the healthy eating habits and nutritional status of school-aged students. A total of 416 children and adolescents between 7 and 14 years of age who were enrolled in 2 complementary public schools were evaluated. A 10month intervention in one of the schools was based on a nutritional education program targeting the students, legal guardians and school staff and involved substantial changes in the school meal menu. Weight, height, BMI, lean mass and body fat mass were evaluated in both the intervention (school 1) and the control (school 2) groups. At the beginning of the study, both school 1 and 2 exhibited a prevalence of overweight $(34.0 \%$ and $28 \%$, respectively) and obese students $(16.9 \%$ and $10.4 \%$, respectively); obesity was significantly reduced by the end of the study $(p=0.001)$. The growth of the students was considered normal for the age range in both schools, although it was slightly larger in school $1(p=0.04)$. Measurements of body composition, which were collected using bioelectrical impedance, indicated an increase in lean mass in both schools $(p<0.01)$ with an increase in body fat mass being observed only in school $2(p=0.01)$. These results, which are related to a significant reduction of the BMI, a change in bodily proportions and an increase in height growth for the students of school 1, indicate the beneficial aspects of the adopted diet.
\end{abstract}

Key words: school meals, nutritional education, body composition in children and adolescents.

1 MD PhD - Children's Institute and Department of Pediatric Medicine. Faculty of Medicine of University of São Paulo

2 MD PhD - Department of Maternal and Infant Health, Faculty of Public Health, University of São Paulo

3 Dietitian - Industrial Social Service of São Paulo State - Quality of Life Department Solidarity and Cultural and Social Development Fund of the State of São Paulo

4 MD PhD - Continuing Education Program of the Oswaldo Cruz Alemão Hospital (Unit Mooca), São Paulo

5 Psychologist MSc PhD - Educational Advising. United Metropolitan Colleges - FMU.

Corresponding author: rfeferbaum@uol.com.br

Suggested citation: Feferbaun R et al. A 10-month anthropometric and bioimpedance evaluation of a nutritional education program for 7 - to 14-year-old students. J. Hum. Growth Dev. 2012; 22(3): 283-290

Manuscript submitted Aug 08 2011, accepted for publication Jul 102012. 


\section{INTRODUÇÃO}

A transição nutricional, observada desde a década de 70 nos países desenvolvidos vem afetando progressivamente aos emergentes de maneira acelerada ${ }^{1,2}$, fazendo com que a prevalência mundial de obesidade na infância apresente rápido crescimento, associando-se a várias complicações, tanto na infância e na adolescência, quanto na fase adulta, adquirindo as características de uma verdadeira epidemia mundial ${ }^{3,4}$.

Vários estudos têm demonstrado que o tempo de duração da obesidade está diretamente associado à morbimortalidade por doenças cardiovasculares $^{3}$. Assim, o fato de estar-se iniciando em idades cada vez mais precoces, inclusive em populações de menor poder aquisitivo ${ }^{5}$, torna a situação ainda mais grave.

Campanhas educativas fundamentadas em maior informação nutricional, não têm demonstrado infelizmente correlação positiva com melhora dos hábitos alimentares. As práticas de consumo alimentar demonstram que a manutenção da saúde não é a única motivação para alimentar-se. Existem diversos fatores que influenciam, como: as preferências individuais, hábitos adquiridos, condicionamento de horários, recursos financeiros, maior ou menor importância concedida a satisfazer as apetências ou requerimentos dos diferentes integrantes do lar, entre outros ${ }^{6}$. Ignorar estas especificidades é de alguma forma condenar ao fracasso qualquer campanha educativa de caráter nutricional. Até o presente momento, as medidas adotadas pelos governos para tentar mudar os hábitos alimentares da população, não têm sido eficientes. Por esta razão, antes de pensar em como mudar os mesmos, deve-se conhecer a natureza do comportamento nutricional da população a ser considerada, e só depois, poder-seá planejar o melhor método que permita favorecer o desenvolvimento de práticas apropriadas para os indivíduos em questão, num momento histórico e cultural determinado.

Considerando crianças brasileiras de 7 a 14 anos, que realizam ao menos uma refeição diária no período escolar, a relevância do papel desempenhado pelas escolas na promoção da saúde e dos bons hábitos alimentares pode ser fundamental, merecendo, portanto, intervenções individualizadas, orientadas a evitar ou corrigir distúrbios nutricionais, a fim de garantir o normal desenvolvimento e crescimento dessa população.

Assim, o objetivo é avaliar um programa de educação nutricional dirigido a alunos, que frequentam escola pública, com o intuito de promover práticas alimentares saudáveis e analisar e comparar as alterações do estado nutricional (antropométrico e dietético) dos respectivos alunos.

\section{MÉTODO}

Trata-se de um estudo de intervenção, prospectivo e controlado. A população estudada com- preendeu 416 crianças e adolescentes, na faixa etária de 7 a 14 anos, de ambos os sexos que frequentam duas escolas públicas de educação complementar ao ensino regular, situadas no centro da cidade de São Paulo.

A população de estudo foi dividida em dois grupos: intervenção (escola 1: 213 alunos) e controle (escola 2: 203 alunos). Os alunos não apresentaram restrições dietéticas ou de atividade física na sua inclusão.

Por um período de 10 meses consecutivos, o programa de intervenção nutricional na escola 2 foi estruturado por meio da presença permanente de uma equipe especializada em educação nutricional para crianças, com participação efetiva dos educadores da escola.

O programa procurou sensibilizar e conscientizar os alunos, bem como os seus responsáveis e educadores da escola, na promoção de hábitos alimentares saudáveis, por meio de reuniões, palestras, oficinas culinárias, aulas práticas e atividades lúdicas, de acordo com a faixa etária considerada.

A intervenção utilizou como modelo o Programa Alimente-se Bem do Sesi-SP7. As preparações culinárias seguiram os parâmetros nutricionais contemplados no referido programa.

Os alunos foram acompanhados diariamente nos horários das refeições, experimentando novas preparações com o aproveitamento integral de alimentos, além das habitualmente consumidas.

Para o cálculo dos cardápios, utilizou-se o programa de software Avanutri ${ }^{8}$. Os cardápios foram elaborados com a finalidade de atender de $40 \%$ a $50 \%$ dos valores de referências de ingestão de nutrientes: valor energético total (VET), carboidrato, proteína, lipídeo, fibras ${ }^{9,10}$, cálcio, ferro e vitaminas ${ }^{11}$.

As duas escolas forneciam diariamente uma alimentação saudável, porém, na escola de intervenção, o programa educativo ofereceu uma maior variedade de hortaliças e frutas, sendo calculado o desperdício por meio do resíduo do resto ingesta. Os alunos tiveram opções de escolhas mais conscientes quanto à quantidade e variedade dos alimentos, visto que, a forma de distribuição da refeição utilizada foi "self-service", ao contrário da escola controle, cuja distribuição da refeição foi feita por sistema de porcionamento, impossibilitando os alunos a fazer voluntariamente suas escolhas alimentares.

Ao início e final do estudo foram realizadas, com os alunos de ambas as escolas, as medidas de peso e estatura, por meio de técnica padronizada de referência ${ }^{12,13}$.

O estado nutricional dos alunos foi avaliado pela determinação do Z - escore para os índices peso/idade, estatura/idade, peso/estatura e IMC, de acordo com os valores de referência da Organização Mundial da Saúde ${ }^{14}$, para cada sexo. Especificamente para a o cálculo dos escores $Z$ de peso recorreu-se ao referencial do CDC $2000^{15}$. Foram considerados com excesso de peso (sobre- 
peso e obesidade) os alunos com IMC igual ou superior a 1 escore Z. A evolução do estado nutricional foi analisada considerando-se as mudanças nos valores do escore $Z$ dos parâmetros antropométricos avaliados.

Nos tempos estabelecidos foram obtidos os valores de massa magra e massa gorda, obtidos a partir de Aparelho Vetorial de Impedância - Sistema BIA, de oito pontos, modelo InBoddy 230, da marca Biospace CO Ltda, e comparadas sequencialmente.

O projeto foi aprovado pelo Comitê de Ética em Pesquisa do Hospital Infantil Darcy Vargas. Os pais ou responsáveis pelas crianças preencheram o
Termo de Consentimento Livre e Esclarecido autorizando-os a fazer parte do estudo, com assentimento dos participantes.

\section{RESULTADOS}

Dados relativos à distribuição de idade, sexo e permanência no estudo estão representados na tabela 1 , observa-se semelhança quanto à distribuição por sexo e idade entre os grupos de crianças de cada escola. Quanto à estatura e ao IMC, não houve diferença estatística nos valores médios observados em ambas as escolas.

Tabela 1: Características dos alunos de ambas as escolas, quanto à distribuição por sexo e idade, no início do estudo e que permaneceram ao longo do trabalho

\begin{tabular}{|c|c|c|c|c|c|}
\hline \multirow[t]{3}{*}{ Sexo } & \multicolumn{2}{|c|}{$\begin{array}{l}\text { Escola } 1 \text { (intervenção) } \\
\qquad(n=206)\end{array}$} & \multicolumn{2}{|c|}{$\begin{array}{c}\text { Escola } 2 \text { (controle) } \\
(n=182)\end{array}$} & \multirow[b]{2}{*}{$\mathrm{p}=0.18^{(N \mathrm{Nig})^{*}}$} \\
\hline & $\begin{array}{c}\text { Masc. } \\
96(46.6 \%)\end{array}$ & $\begin{array}{c}\text { Masc. } \\
110(53.4 \%)\end{array}$ & $\begin{array}{c}\text { Masc. } \\
98(53.8 \%)\end{array}$ & $\begin{array}{c}\text { Feminino } \\
110(53.4 \%)\end{array}$ & \\
\hline & \multicolumn{2}{|c|}{ Média \pm desvio padrão } & \multicolumn{2}{|c|}{ Média \pm desvio padrão } & \\
\hline Idade (anos) & 10,00 & $\pm 1,80$ & 10,10 & $\pm 1,90$ & $\mathrm{p}=0,14^{(N S i g)^{* *}}$ \\
\hline Estatura (escore Z) & 0,11 & $\pm 1,06$ & 0,03 & $\pm 0,99$ & $\mathrm{p}=0,73^{(\mathrm{NSig}) * *}$ \\
\hline IMC (escore Z) & 0,11 & $\pm 1,06$ & 0,41 & $\pm 1,19$ & $\mathrm{p}=0,29^{(N S i g) * *}$ \\
\hline
\end{tabular}

Como se observa na tabela 1 , o crescimento estatural é compatível com o referencial da $\mathrm{OMS}^{14}$. Quanto ao IMC, revela-se que há uma tendência ao sobrepeso em ambas às escolas, quando compara- da ao mesmo referencial.

No início do estudo, se encontrou presença de excesso de peso em ambas as escolas (tabela 2).

Tabela 2: Frequências de distúrbios nutricionais dos alunos de ambas as escolas, no início e no final do programa, diagnosticados por critérios antropométricos de índice de massa corpórea - IMC*

\begin{tabular}{|c|c|c|c|c|}
\hline & \multicolumn{2}{|c|}{ Escola 1} & \multicolumn{2}{|c|}{ Escola 2} \\
\hline & Início & Fim & Início & Fim \\
\hline Magreza & $2,9 \%$ & $3,4 \%$ & $1,6 \%$ & $1,6 \%$ \\
\hline Sobrepeso & $17,5 \%$ & $18,1 \%$ & $17,6 \%$ & $16,5 \%$ \\
\hline Obesidade & $16,9 \%$ & $13,1 \%$ & $10,4 \%$ & $10,4 \%$ \\
\hline
\end{tabular}

* De acordo com os critérios propostos pelo Ministério da Saúde, Coordenação-Geral da Política de Alimentação e Nutrição - CGPAN, SISVAN - Sistema de Vigilância Alimentar e Nutricional (http://nutricao.saude.gov.br/sisvan. php?conteudo=curvas_cresc_oms).

Tabela 3: Média de escore Z de estatura, peso e IMC dos alunos ao início e ao término do estudo, segundo a escola que frequentavam

\begin{tabular}{|c|c|c|c|c|c|c|}
\hline \multirow{2}{*}{$\begin{array}{c}\text { Variáveis } \\
\text { (Média } \pm \mathrm{dp} \\
\text { de escore } Z \text { ) }\end{array}$} & \multicolumn{2}{|c|}{$\begin{array}{c}\text { Escola } 1 \\
(n=206)\end{array}$} & \multicolumn{4}{|c|}{$\begin{array}{c}\text { Escola } 2 \\
(n=182)\end{array}$} \\
\hline & Inicial & Final & & Inicial & Final & \\
\hline Estatura & $\begin{array}{c}0,11 \\
\pm 1,05\end{array}$ & $\begin{array}{c}0,14 \\
\pm 1,04\end{array}$ & $\mathrm{p}=0,04^{*(* *)}$ & $\begin{array}{c}0,03 \\
\pm 0,99\end{array}$ & $\begin{array}{c}0,05 \\
\pm 0,97\end{array}$ & $p=0,27^{*}$ \\
\hline Peso** & $\begin{array}{c}0,24 \\
\pm 1,21\end{array}$ & $\begin{array}{c}0,21 \\
\pm 1,18\end{array}$ & $p=0,20^{*}$ & $\begin{array}{c}0,14 \\
\pm 1,04\end{array}$ & $\begin{array}{l}-0,03 \\
\pm 2,30\end{array}$ & $p=0,30^{*}$ \\
\hline IMC & $\begin{array}{c}0,53 \\
\pm 1,05\end{array}$ & $\begin{array}{c}0,45 \\
\pm 1,04\end{array}$ & $\mathrm{p}=0,01^{*(* *)}$ & $\begin{array}{c}0,39 \\
\pm 1,18\end{array}$ & $\begin{array}{c}0,36 \\
\pm 1,19\end{array}$ & $p=0,24^{*}$ \\
\hline
\end{tabular}

* Teste de Student para variáveis emparelhadas;

** Referencial do Center for Diseases Control - EUA;

*** Significância $\mathrm{P}<0,05$ 
A Tabela 3 demonstra os resultados antropométricos no início e no final do estudo em ambas as escolas. Nota-se que houve crescimento adequado em relação ao peso e estatura, sendo que os alunos da escola 1 tiveram um crescimento estatural estatisticamente significante, não registrado na escola 2.

Tabela 4: Diferença média entre o escore $Z$ de IMC dos alunos que apresentavam excesso de peso (OMS, 2007), ao início e ao término do estudo, segundo a escola que frequentavam

$\begin{array}{cccc} & \text { Escola 1 } & \text { Escola 2 } & \\ \text { Média } \pm \text { dp } & -0,242 \pm 0,053 & -0,101 \pm 0,054 & p<0,03^{*(* *)} \\ \text { da diferença de IMC } & (n=71) & (n=50) & \end{array}$

* Teste de Student para variáveis emparelhadas

*** Significante

A análise da composição corpórea das crianças por meio da BIA, demonstrada na tabela 3, mostra um aumento significante de massa muscular em ambos os grupos. Com relação à massa gor- da, os dados da tabela evidenciam que, enquanto os alunos da escola 2 aumentaram a porcentagem de massa gorda, o mesmo não ocorreu com os alunos da escola 1.

Tabela 5: Médias de Massa Muscular Esquelética (MME) e Massa Gorda Corpórea (MG), obtidas por impedância bioelétrica, dos alunos ao início e ao término do estudo, segundo a escola que frequentavam

\begin{tabular}{|c|c|c|c|}
\hline & & Esola $1(n=206)$ & Escola $2(n=182)$ \\
\hline MME (média \pm dp) & $\begin{array}{c}\text { Inicial } \\
\text { Final } \\
\left.\mathrm{p}<0,01^{* * *}\right)\end{array}$ & $\begin{array}{l}14,3 \pm 4,68 \\
15,6 \pm 4,98 \\
p<0,01^{*(* *)}\end{array}$ & $\begin{array}{l}14,7 \pm 4,46 \\
15,8 \pm 4,87\end{array}$ \\
\hline MG (média \pm dp) & $\begin{array}{l}\text { Inicial } \\
\text { Final }\end{array}$ & $\begin{array}{l}22,9 \pm 9,31 \\
23,1 \pm 9,04 \\
p=0,20^{*} \text { (NSig) }\end{array}$ & $\begin{array}{l}21,2 \pm 8,86 \\
22,2 \pm 9,54 \\
p=0,01^{*(* *)}\end{array}$ \\
\hline
\end{tabular}

* Teste de Student para variáveis emparelhadas

*** Significante

Com relação à alimentação, observou-se que a escola 1 teve um aumento de $30 \%$ no consumo de frutas, verduras e legumes, com $95 \%$ de aceitação dos mesmos, além da diminuição do lixo orgânico em 33\%.

Notou-se igualmente envolvimento das famílias nos encontros agendados, com mobilização de todos os responsáveis pelos alunos.

\section{DISCUSSÃO}

A transição entre a infância e a adolescência é uma etapa crucial no desenvolvimento humano e implica no aparecimento de múltiplas mudanças fisiológicas e psicológicas. Umas e outras influenciam diretamente no comportamento, nas necessidades nutricionais e nos hábitos alimentares. Associa-se também a eclosão hormonal responsável por importantes mudanças na composição corpórea do individuo. Em consequência, os requerimentos de energia e nutrientes vão a ser superiores em relação a outras etapas da vida ${ }^{16,17}$.

As mudanças sociais e culturais, próprias da nossa época, tendem a afetar os padrões dietéticos e a atividade física, sendo estes cada vez mais dependentes do individuo em formação. Isto pode conduzir à aquisição de hábitos inadequados que, na maioria dos casos, se mantém ao longo da vida adulta, com o conseguinte risco para a saúde. É comprovado que a presença de distúrbios nutricionais, originados durante a infância e adolescência, incrementam de forma notável a probabilidade de algumas doenças como: dislipidemia/aterosclerose $^{18}$, transtornos do comportamento alimentar, obesidade/diabete ${ }^{19}$, osteoporose ${ }^{20}$, e certos tipos de cânceres ${ }^{21}$, entre outras.

A iniciativa do presente trabalho surge ao registrar a problemática da população infantil brasileira decorrente do consumo inadequado de alimentos, associada fundamentalmente a mudanças do comportamento alimentar e à inatividade física. Pesquisa de Orçamento Familiar ${ }^{22}$, realizada entre 2008/2009 pelo Instituto Brasileiro de Geografia e Estatística, mostra que uma em cada três crianças, com idade entre cinco e nove anos, está com peso acima do recomendado pela Organização Mundial da Saúde ${ }^{23}$. O índice de jovens de 10 a 19 anos com excesso de peso passou de 3,7\%, em 1970, para 21,7\%, em 2009.

Embora esta problemática não seja exclusiva do Brasil, não existem infelizmente dados comparáveis sobre a ingestão de alimentos e nutrientes, na maior parte dos países da comunidade europeia e dos EE.UU., apesar das alarmantes cifras registradas. A falta de estudos multicêntricos, com casuísticas numerosas e metodologia unificada, dificulta ainda mais a elaboração de propostas de 
promoção da saúde baseadas em evidências. Por outro lado, a avaliação quantitativa do estado nutricional em crianças e adolescentes, torna-se muitas vezes difícil pela falta de uma definição consensada de obesidade infantil, no entanto a $\mathrm{OMS}^{14}$ na tentativa de contemplar e unificar estes aspectos preconiza a utilização das curvas de crescimento, recomendando como pontos de corte para o diagnóstico de obesidade os percentuais P85 e P95 do IMC, respectivamente.

Utilizando o Índice de Massa Corporal (IMC), em populações estatisticamente homogêneas (tabela 1), como indicador quantitativo do estado nutricional verificamos que o valor médio registrado em ambas as escolas exibia tendência para o sobrepeso, estando em torno de 0,5 desvio-padrão (dp) acima da mediana preconizada pela $\mathrm{OMS}^{14}$. Este dado é compatível com o registrado em crianças em idade escolar no país, em decorrência da transição nutricional que vem ocorrendo e que é reflexo da tendência mundial constatada nos países industrializados desde o começo da década dos $80^{1,24}$.

Ao início do estudo nas escolas 1 e 2 verificou-se respectivamente frequências de excesso de peso de 34,4 e 28,0\%, e de obesidade de 16,9 e $10,4 \%$ (tabela 2). Embora os números absolutos ao final do estudo sugiram uma tendência de redução, não se observou variação estatisticamente significante nestas proporções. No entanto, quando se consideram as crianças obesas da escola 1 , demonstra-se redução do grau de obesidade ao final do período de intervenção. Este resultado, aparentemente pouco impactante sobre a proporção de crianças com excesso de peso pode, em tese, ser atribuído ao curto tempo de duração da intervenção. Entretanto é importante salientar que a mesma permitiu a modificação de parâmetros antropométricos, com particular destaque para a diminuição significativa do valor médio do escore z de IMC (tabelas 1 e 2 ) das crianças que mais necessitavam melhorar sua condição nutricional, isto é, os obesos.

Existem numerosos estudos clínicos aleatórios publicados sobre a prevenção da obesidade infanto-juvenil no âmbito escolar, com limitações metodológicas importantes, assim como numerosas revisões ${ }^{25-30}$ e alguns guias de prática clínica ${ }^{31-}$ 33. Por outro lado, estes estudos mostram importante heterogeneidade em quanto às estratégias desenvolvidas (dieta, atividade física), ao local de atuação (escola, trabalho, seio familiar) e à implicação da família no processo. Fundamentalmente no que diz respeito aos seus resultados, mesmo sem se considerar o tempo de intervenção, não é fácil o estabelecimento de uma nítida associação do tipo causa-efeito.

Dentre as intervenções dietéticas para prevenir a obesidade, com extensão de um ano ou mais de duração, especialmente destinadas a crianças e adolescentes, alguns trabalhos mostraram resultados satisfatórios como o Planet Health ${ }^{34}$, realizado em 10 escolas dos EE.UU., incluindo 1.295 escolares, de 11 a 12 anos. A intervenção, orientada a mudanças na dieta e redução dos comportamentos sedentários, evidenciou diminuição significativa da obesidade no grupo de intervenção, com prevalência da mesma no grupo controle. Por sua vez, o estudo APPLES (Active Programme Promoting Lifestyle in Schools), que teve lugar na Inglaterra, envolvendo 634 escolares, de 7 a 11 anos, não mostrou diferenças estatísticas nos valores do IMC, pré e pós-intervenção, apesar de implementar ações similares, com igual tempo de duração ${ }^{35}$. Intervenções nutricionais mais extensas, de até 2 anos, também mostraram-se ineficazes ${ }^{36}$.

Dos poucos trabalhos realizados em crianças e adolescentes com duração menor de um ano, destaca-se um estudo conduzido no Chile, de seis meses de duração, que teve por objetivos melhorar a educação nutricional e reforçar a atividade física em 2.375 crianças de escolas do ensino primário ${ }^{37}$. Na avaliação ao final da intervenção, o IMC não foi significativamente diferente entre os grupos de intervenção e controle.

Estes resultados de alguma forma reforçam o conceito de que o tempo de atuação "per se" não garante o êxito esperado, apesar de dispormos de dietas padronizadas e atividade física regular, ficando os resultados na dependência de outros facilitadores além do próprio indivíduo.

Um dos fatores facilitadores na redução percentual do IMC nas crianças com excesso de peso, em um tempo relativamente curto de 8 meses como o aqui apresentado, provavelmente deva-se ao processo de sensibilização realizado, envolvendo além dos alunos, os seus pais e os educadores. Diferentes intervenções podem ter efeito positivo, embora pequeno no consumo de frutas e verduras, quando direcionadas a meninos e meninas indistintamente. Os maiores efeitos associam-se às intervenções que envolvem os progenitores e, também, os professores diretamente vinculados à criança ${ }^{38}$.

Em relação à prática de atividade física, os programas desenvolvidos em crianças e adolescentes para tratamento do sobrepeso e da obesidade, não se têm mostrado sempre efetivos na redução do IMC $^{39}$. Contudo, é reconhecido o fato de que a atividade física pode diminuir temporalmente o percentual de gordura corporal ${ }^{40,41} \mathrm{e}$, consequentemente, a presença de fatores de risco para as doenças cardiovasculares ${ }^{42,43}$

No presente estudo, não foram incluídas crianças com doenças orgânicas prévias e ou necessidades dietéticas especiais. Todas elas demonstram um bom desempenho físico e do estado geral, pelo que não foram indicadas atividades físicas complementares nem restrições dietéticas específicas.

Um dos diferenciais aportados por este estudo em relação aos trabalhos antes comentados foi à possibilidade que os escolares tiveram de escoIher os alimentos de acordo com as suas preferências, o bom "gosto" por eles registrado, tornando os mesmos convidativos e permitindo-Ihes exercer 
o prazer da autonomia em relação às refeições. 0 aumento da oferta, a variedade de apetites contemplados e de alimentos saudáveis e acessíveis ao universo escolar e familiar, possibilitou que os alunos pudessem dispor na escola 1 de opções diferentes e saborosas, sendo parte das mesmas reproduzidas nos respectivos lares mediante envolvimento dos progenitores. De alguma forma, intervenções efetivas parecem ser aquelas que não colocam a atenção nos aspectos meramente das necessidades de nutrientes necessários para o equilíbrio saúde/doença e sim no prazer que oferece a comida. Isto viabiliza uma maior adesão ao consumo de frutas, verduras e legumes de estação, com aproveitamento integral de seus componentes, fidelizando o consumidor primário que é o aluno e, secundariamente, a sua família.

Em relação ao crescimento dos alunos, este foi considerado normal para a faixa etária. Destacou-se que na escola 1, objeto da intervenção, foi significativamente superior (tabela 1 ). A alimentação oferecida quase certamente foi um fator influenciador positivo para o padrão de altura atingido. Nesse sentido, a correção de hábitos nutricionais na criança, mediante adoção de uma dieta equilibrada, garante, na maioria das vezes o seu normal desenvolvimento.

Embora o peso para a idade não seja considerado um parâmetro importante para se avaliar o estado nutricional de crianças acima de 5 anos de idade $^{44}$, os dados obtidos mostraram que não houve alterações significantes estatisticamente nas médias de peso observadas em ambas as escolas (tabela 1). Mas, em relação aos resultados da composição corporal, obtidos a partir da impedância bioelétrica, se observou em ambas as escolas um aumento da massa magra dos escolares que, no entanto, foi acompanhado de um aumento significativo da massa gorda apenas na escola controle (tabela3). Não ter havido aumento de massa gor-

\section{REFERÊNCIAS}

1. Popkin BM. The obesity epidemic is a worldwide phenomenon. Nutr Rev. 1998;56(4):106-14.

2. Popkin BM. The nutrition transition and its health implications in lower income countries. Public Health Nutr. 1998;1(1):5-21.

3. Oliveira $C L$, Fisberg M. Obesidade na infância e adolescência - Uma verdadeira epidemia [Childhood and adolescence obesity - a real epidemic]. Arq Bras Endocrinol Metab. 2003;47(2):107-8.

4. Mello ED, Luft VC, Meyer F. Obesidade infantil: como poderemos ser eficazes? [Childhood obesity: toward effectiveness?] J Pediatr (Rio J). $2004 ; 80(3): 173-82$. da nas crianças da escola objeto da intervenção nutricional reforça a existência de um impacto positivo da mesma. Estes resultados, mormente se correlacionados à redução significativa do IMC, às proporções corporais, e ao crescimento estatural atingido, ratificam que a alimentação adotada foi de fato benéfica, os quais subsidiaram a implantação do Programa Consciência Alimentar, na Rede Municipal Escolar do Estado de São Paulo45,46.

Assim, estes resultados direcionam para a necessidade de continuar realizando novos ensaios clínicos. Assim será possível evoluir no aperfeiçoamento de uma forma de intervenção mais efetiva para a prevenção do sobrepeso e a obesidade na infância, bem como testar a eficácia de intervenções breves na atenção primária e na elaboração da merenda escolar, visando estabelecer o hábito de se consumir uma alimentação saudável. É importante ressaltar, que para que crianças e adolescentes alcancem uma mudança de vida por meio da educação nutricional integrada a outras ações, como a prática de atividade física, o processo de intervenção precisa ser contínuo e iniciado em idade precoce. Sem a adoção de medidas dietéticas e a implementação de mudanças no estilo de vida da criança e de sua familia, as chances de êxito, em termos de diminuir as morbidades que decorrem do sobrepeso e obesidade no início da idade adulta, serão certamente reduzidas.

\section{Agradecimentos}

Tereza Toshiko Watanabe (in memorian): Divisão de Nutrição e Responsabilidade Social do Serviço Social da Indústria (SESI) - Departamento Regional de São Paulo, Luiz Roberto Barradas Barata (in memorian): Secretaria da Saúde do Estado de São Paulo-SP, Sergio Antonio Bastos Sarrubbo: Hospital Infantil Darcy Vargas, São Paulo-SP.

5. Nascimento VG, Schoeps DO, Souza SB, Souza JMP, Leone C. Risk of overweight and obesity in preschoolers attending private and philanthropic schools. Rev Assoc Med Bras. 2011;57(6):643-7.

6. McDougall, P. Teenagers and nutrition: assessing the levels of knowledge. Health Visit. 2004;71(2):62-4.

7. Serviço Social da Indústria (SESI), Departamento Regional de São Paulo. Alimentese bem: 200 receitas econômicas e nutritivas. $12^{a}$ ed. [Industrial Social Service (SESI), São Paulo Regional Department. Eat well: 200 inexpensive and nourishing recipes. 12th ed]. São Paulo: SESI-SP; 2008.

8. Santana RI. Avanutri sostware de avaliação e prescrição nutricional [Avanutri nutritional 
evaluation and prescription software]. Rio de Janeiro version $4.0 ; 2009$.

9. FAO/WHO/UNU. Human energy requirements. Report of a joint FAO/WHO/UNU expert consultation. Rome: Food and Agriculture Organization; 2001.

10. World Health Organization/Food and Agriculture Organization. Joint report of expert consultation. Diet, nutrition and the prevention of chronic diseases. Geneva: World Health Organization; 2003. (WHO Technical Report Series; 916).

11. Institute of Medicine. Dietary reference intake for energy, carbohydrate, fiber, fat, body fat acids, cholesterol, protein, and amino acids. Washington, DC: National Academy Press; 2005.

12. Frisancho R. Anthropometric standards for the assessment of growth and nutritional status. Ann Arbor: The University of Michigan Press; 1999.

13. Organización Panamericana de la Salud. Conjunto de acciones para la reducción multifactorial de enfermedades no transmisibles [Actions for multifactorial non-transmissible disease reduction], Washington, DC: OPS; 1997.

14. WHO. Growth reference data for 5-19 years, 2007. Available from: http://www.who.int/ growthref/en/.

15. National Center for Health Statistics, Centers for Disease Control and Prevention [homepage on the Internet]. Atlanta, GA: Department of Health and Human Services; [updated May 20, 2004; cited 2004 November 4]. 2000 CDC Growth Charts: United States; [about 3 screens]. Available from: http://www.cdc.gov/ growthcharts.

16. FAO/WHO/ONU. Expert consultation report. Energy and protein requirements. Geneva: WHO; 1985. (Technical Report Series; 724).

17. Southon S, Wright AJA, Finglas PM, Bailey AH, Loughridge JM, Walker AD. Dietary intake and micronutrient status of adolescents: effect of vitamin and trace element supplementation on indices of status and performance of verbal and nonverbal intelligence. $\mathrm{Br} \mathrm{J}$ Nutr. 1994;71(6):897-987.

18. Csábi G, Török K, Jeges S, Molnár D. Presence of metabolic cardiovascular syndrome in obese children. Eur J Pediatr. 2000;159(1-2):91-4.

19. Marcos A, Montero A, López-Varela S, Morandé G. Eating disorders (obesity, anorexia and bulimia), immunity and infection. In: Tontisirin K, Susking $R$, editors. Nutrition, immunity and infection disease in infants and children. 45th Nestle Nutrition Workshop. Bangkok, Thailand. Vevey. Nestle Nutrition Services. 2001;45:243-79.

20. Molgaard C, Thomsen BL, Michaelsen KF. Influence of weight, age and puberty on bone mineral content in healthy children and adolescents. Acta Paediatr. 1998;87(5):494-9.
21. Frankel S, Gunnell DJ, Peters TJ, Maynard M, Davey Smith G. Childhood energy intake and adult mortality from cancer: the Boyd Orr Cohort Study. BMJ. 1998;316(7130):499-504.

22. Pesquisa de orçamentos familiares 2008-2009. Informe. Quarto Fórum SIPD [2008-2009 family budget survey]. Report. Fourth SIPD Forum. Rio de Janeiro, 2008. p.24.

23. WHO Multicentre Growth Reference Study Group. Breastfeeding in the WHO Multicentre Reference Study. Acta Paediatr. 2006; (Suppl 450): 16-26.

24. Flegal KM, Carroll MD, Ogden CL, Johnson CL. Prevalence and trends in obesity among US adults, 1999-2000. JAMA. 2002;288(14):1723-7.

25. Flynn MA, McNeil DA, Maloff $B$, Mutasingwa D, Wu $M$, Ford $C$, et al. Reducing obesity and related chronic disease risk in children and youth: a synthesis of evidence with 'best practice' recommendations. Obes Rev. 2006;7(Suppl 1):7-66.

26. Connelly JB, Duaso MJ, Butler G. A systematic review of controlled trials of interventions to prevent childhood obesity and overweight: a realistic synthesis of the evidence. Public Health. 2007;121(7):510-7.

27. Brown T, Summerbell C. Systematic review of school-based interventions that focus on changing dietary intake and physical activity levels to prevent childhood obesity: an update to the obesity guidance produced by the National Institute of Health and Clinical Excellence. Obes Rev. 2009;10(1):110-41.

28. Kamath CC, Vickers KS, Ehrlich A, McGovern L, Johnson J, Singhal $V$ et al. Behavioral interventions to prevent childhood obesity. A systematic review and meta-analyses of randomized trials. J Clin Endocrinol Metab. 2008;93 (12):4606-15.

29. Shaya FT, Flores D, Gbarayor CM, Wang J. School-based obesity interventions: a literature review. J Sch Health. 2008;78(4):189-96.

30. Wofford LG. Systematic review of childhood obesity prevention. J Pediatr Nurs. 2008;23(1):5-19.

31. National Institute for Health and Clinical Excellence. Obesity. Guidance on the prevention, identification, assessment and management of overweight and obesity in adults and children. NICE Clinical Guideline 43. December 2006.

32. Lau DC, Douketis JD, Morrison KM, Hramiak IM, Sharma AM, Ur E. 2006 Canadian clinical practice guidelines on the management and prevention of obesity in adults and children. CMAJ. 2007;176(8 Suppl):1-13.

33. Han JC, Lawlor DA, Kimm SYS. Childhood obesity. Progress and challenges. Lancet. 2010;375(9727):1737-48.

34. Gortmaker SL, Peterson K, Wiecha J, Sobal AM, Dixit S, Fox MK et al. Reducing obesity via a school-based interdisciplinary intervention 
among youth. Arch Pediatr Adolesc Med. 1999;153(4):409-18.

35. Sahota P, Rudolf MCJ, Dixey R, Hill AJ, Barth $\mathrm{JH}$, Cade J. Randomized controlled trial of primary school based intervention to reduce risk factors for obesity. BMJ. 2001; 323(7320): 1029-32.

36. Donnelly JE, Jacobsen DJ, Whatley JE, Hill JO, Swift LL, Cherrington $A$ et al. Nutrition and physical activity program to attenuate obesity and promote physical and metabolic fitness in elementary school children. Obes Res. $1996 ; 4(3): 229-43$

37. Kain J, Uauy R, Albala, Vio F, Cerda R, Leyton B. School-based obesity prevention in Chilean primary school children: methodology and evaluation of a controlled study. Int J Obes. 2004;28(4):483-93.

38. Thomas J, Sutcliffe K, Harden A, Oakley A, Oliver S, Rees R, et al. Children and healthy eating: a systematic review of barriers and facilitators. London: EPPI Centre, Social Science Research Unit, Institute of Education, University of London; 2003.

39. Daley AJ, Copeland RJ, Wright NP, Roalfe A, Wales $\mathrm{JKH}$. Exercise therapy as a treatment for psychopathologic conditions in obese and morbidly obese adolescents: a randomized, controlled trial. Pediatrics. 2006;118(5):2126-34.

40. Atlantis E, Barnes EH, Fiatarone Singh MA. Efficacy of exercise for treating overweight in children and adolescents: a systematic review. Int J Obes (Lond). 2006;30(7):1027-40.

41. Carrel AL, Clark RR, Peterson S, Nemet BA, Sullivan J, Allen DB. Improvement of fitness, body composition, and insulin sensitivity in overweight children in a school-based exercise program: a randomized, controlled study. Arch Pediatr Adolesc Med. 2005;159(10):963-8.

42. Allen DB, Nemeth BA, Clark RR, Peterson SE, Eickhoff J, Carrel AL. Fitness is a stronger predictor of fasting insulin levels than fatness in overweight male middle-school children. ] Pediatr. 2007;150(4):383-7.

43. Eisenmann JC, Welk GJ, Wickel EE, Blair SN. Combined influence of cardiorespiratory fitness and body mass index on cardiovascular risk factors among 8-18 year old youth: The Aerobics Center Longitudinal Study. Int J Pediatr Obes. 2007;2(2):66-72.

44. WHO Multicentre Growth Reference Study Group. WHO Child Growth Standards based on length/height, weight and age. Acta Paediatr. 2006;(Suppl 450):76-85.

45. Fundo Social de Solidariedade do Estado de São Paulo. Available from: http:// www.fundosocial.sp.gov.br/portal.php/sobregalerias

46. Decreto No 55.620, de 24 de Março de 2010. Available from: http://www.al.sp.gov.br/ repositorio/legislacao/decreto/2010/ d e creto\% 20 n. 55.620 , \% 20 d e \% 2024.03.2010.htm 